\title{
Arsenic methylation by an arsenite S-adenosylmethionine methyltransferase from Spirulina platensis
}

\author{
Yuqing Guo ${ }^{1,2}$, Ximei Xue $e^{2}$, Yu Yan², Yongguan $\mathrm{Zhu}^{2,3}$, Guidi Yang ${ }^{1, *}$, Jun Ye $e^{2, *}$ \\ 1. Fujian Provincial Key Laboratory of Agroecological Processing and Safety Monitoring, College of Life Sciences, Fujian Agriculture and Forestry \\ University, Fuzhou 350002, China. E-mail: yqguo@iue.ac.cn \\ 2. Key Laboratory of Urban Environment and Health, Institute of Urban Environment, Chinese Academy of Sciences, Xiamen 361021, China \\ 3. State Key Laboratory of Urban and Regional Ecology, Research Center for Eco-Environmental Sciences, Chinese Academy of Sciences, \\ Beijing 100085, China
}

\section{A R T I C L E I N F O}

Article history:

Received 23 April 2016

Revised 17 June 2016

Accepted 29 June 2016

Available online 14 July 2016

\section{Keywords:}

Spirulina platensis

Arsenic

Methylation

Methyltransferase

\begin{abstract}
A B S T R A C T
Arsenic-contaminated water is a serious hazard for human health. Plankton plays a critical role in the fate and toxicity of arsenic in water by accumulation and biotransformation. Spirulina platensis (S. platensis), a typical plankton, is often used as a supplement or feed for pharmacy and aquiculture, and may introduce arsenic into the food chain, resulting in a risk to human health. However, there are few studies about how S. platensis biotransforms arsenic. In this study, we investigated arsenic biotransformation by S. platensis. When exposed to arsenite (As(III)), S. platensis accumulated arsenic up to $4.1 \mathrm{mg} / \mathrm{kg}$ dry weight. After exposure to $\mathrm{As}(\mathrm{III})$, arsenate $(\mathrm{As}(\mathrm{V}))$ was the predominant species making up $64 \%$ to $86 \%$ of the total arsenic. Monomethylarsenate $(\mathrm{MMA}(\mathrm{V}))$ and dimethylarsenate (DMA(V)) were also detected. An arsenite S-adenosylmethionine methyltransferase from S. platensis (SpArsM) was identified and characterized. SpArsM showed low identity with other reported ArsM enzymes. The Escherichia coli AW3110 bearing SparsM gene resulted in As(III) methylation and conferring resistance to As(III). The in vitro assay showed that SpArsM exhibited As(III) methylation activity. DMA(V) and a small amount of MMA(V) were detected in the reaction system within $0.5 \mathrm{hr}$. A truncated SpArsM derivative lacking the last 34 residues still had the ability to methylate As(III). The three single mutants of SpArsM (C59S, C186S, and C238S) abolished the capability of As(III) methylation, suggesting the three cysteine residues are involved in catalysis. We propose that SpArsM is responsible for As methylation and detoxification of As(III) and may contribute to As biogeochemistry.

(C) 2016 The Research Center for Eco-Environmental Sciences, Chinese Academy of Sciences.
\end{abstract} Published by Elsevier B.V.

\section{Introduction}

Arsenic (As) is one of the most common contaminants in the environment. The average As concentration in marine waters is around $1.5 \mu \mathrm{g} / \mathrm{L}$ (Cullen and Reimer, 1989). While concentrations of As vary in freshwaters, depending on the geological features and the result of As mobilization through a combination of natural processes as well as through a range of anthropogenic activities (Smedley and Kinniburgh, 2002), As-contaminated water is causing worldwide drinking water safety and food supply problems as around 150 million people are exposed to As-contaminated drinking water, particularly in South or Southeast Asia (Brammer and Ravenscroft, 2009; Christen, 2001; Meliker et al., 2007; Pan et al., 2014). In addition to drinking

\footnotetext{
* Corresponding authors. E-mail addresses: guidiyang@fafu.edu.cn (Guidi Yang), jye@iue.ac.cn (Jun Ye).
} 
water, As biomagnification by alga, fishes, and other aquatic organisms also introduces As into the food chain and can be a potential source of threat to human health (Maher et al., 2011; Rahman et al., 2012).

Arsenic biotransformation by organisms plays an important part in its fate in the environment and toxicity to cells (Ye et al., 2012). Arsenic is primarily present in the form of inorganic As in natural environments, and inorganic As can be biotransformed into organic As, such as methylated arsenicals, arsenosugars, and arsenolipids. The mechanisms of As biotransformation including oxidation, reduction, and methylation have been widely studied. Arsenite (As(III)) oxidation is believed to be a detoxification pathway since arsenate $(\mathrm{As}(\mathrm{V}))$ is less toxic than As(III) (Armendariz et al., 2015; Paez-Espino et al., 2009). Although the possible intermediate products methylarsenite (MMA(III)) and dimethylarsenite (DMA(III)) are more toxic, As methylation is acknowledged as a pathway of detoxification in microbes (Styblo et al., 2002; Wang et al., 2014b). One classical As biomethylation scheme can be summarized as alternating reduction and methylation, producing less toxic methylated arsenicals such as methylarsenate $(\mathrm{MMA}(\mathrm{V}))$, dimethylarsenate (DMA(V)), and trimethylarsine oxide (TMAO(V)) (Bentley and Chasteen, 2002; Challenger, 1945). Recent studies proposed alternative pathways in which MMA(III) and DMA(III) remain trivalent throughout the whole catalytic cycle (Hayakawa et al., 2005; Marapakala et al., 2012).

S. platensis is a cyanobacterium that forms massive populations in freshwater, brackish condition, and in some marine environments (Vonshak, 1997). It has been used as food by the Aztecs in the 16th century (Del Castillo, 1956), and was rediscovered in the mid-1960s (Vonshak, 1997). S. platensis is rich in protein, vitamins, and essential fatty acids (Babadzhanov et al., 2004; Ciferri, 1983), so it is an attractive food source for less-developed countries where people still suffer from famine problems. Nowadays, it is often used as a commercial medicine (as pills, tablets, or capsules) recommended as a supplement of vitamins and proteins (Campanella et al., 1998) or applied as a feed in aquaculture (Vonshak, 1997). In a word, it has a basal position in the food web, and plays an important role in the ecosystem. However, the total As concentration of Spirulina supplement from Mexico and Chad was detected to exceed the maximum limit of the National Health Surveillance Agency of Brazil (Richmond and Hu, 2013). The heavy metal excess of edible Spirulina has attracted increasing attention, and the heavy metal pollution might come from breeding, drying, and processing. At the same time, S. platensis can rapidly accumulate heavy metals, for example the maximum sorption capacity of $\mathrm{As}(\mathrm{V})$ reached up to $525 \mathrm{mg} / \mathrm{g}$ (Doshi et al., 2009). This indicated that S. platensis in the natural environment might be an effective bioremediation material for removing heavy metals from water (Disyawongs, 2002; Doshi et al., 2009; Lodi et al., 2008; Solisio et al., 2006). Therefore, studies about accumulation and biotransformation of As by S. platensis are needed, since only total As was measured in previous studies.

Thus, we investigated the molecular mechanism of As biotransformation by $\mathrm{S}$. platensis by treating it with As(III). Furthermore, a gene encoding the As(III) methyltransferase (SpArsM) was identified, and the SpArsM protein was purified and characterized.

\section{Materials and methods}

\subsection{Organisms and cultivations}

S. platensis FACHB-439 was obtained from the Institute of Hydrobiology, Chinese Academy of Science, Wuhan, China and incubated in modified Zarrouk's medium (Seshadri and Thomas, 1979). The growth condition for S. platensis was under a 12-hr light-dark cycle with a light intensity of approximately $50 \mu \mathrm{mol} /\left(\mathrm{m}^{2} \cdot \mathrm{sec}\right), 26^{\circ} \mathrm{C}$ (day) $/ 25^{\circ} \mathrm{C}$ (night). During incubation, the cultures were shaken by hand twice a day. E. coli cells were grown in Luria-Bertani (LB) medium at $37^{\circ} \mathrm{C}$ with corresponding antibiotics. Strain DH5 $\alpha$ (TransGen) was used for plasmid construction. Strain AW3110 ( $\triangle$ arsRBC) (Carlin et al., 1995) was used for functional verification of the arsM gene. Strain Transetta (DE3) (TransGen) was used for protein expression and purification.

\subsection{Arsenic species in S. platensis}

S. platensis FACHB-439 in the exponential phase were treated with indicated concentrations of As(III) for 15 days. CK treatments ( $1 \mu \mathrm{mol} / \mathrm{L}$ As(III) without bacteria) were placed under the same conditions. Trimethylarsenite (TMA(III)) released from cyanobacteria was collected as reported previously (Yin et al., 2011). As species was determined to investigate biotransformation of As, and the extraction method of cellular As was performed as described before (Yan et al., 2015).

\subsection{Cloning of SparsM from S. platensis}

The genomic DNA of S. platensis FACHB-439 was extracted by using a DNA extraction kit (Bioteke) according to the manufacturer's instructions. As the genome of S. platensis FACHB-439 has not been sequenced, we chose the putative arsM in Arthrospira platensis (GenBank accession number WP_006619316.1) to assist in the design of primers. An 1185-bp fragment was successfully amplified by PCR with primers 5'-CATATGACTCCAGCTAAATC GGCAAATACTGC-3' (NdeI site underlined), and 5'-CTCGAGACA ACAACTTGATGGAGAAC-3' (XhoI site underlined). The PCR product was cloned into the pMD19-T vector (Takara) to create plasmid pMD19-T-SparsM. After extracting with a plasmid extraction mini kit (Tiangen), the plasmid was digested with NdeI and XhoI and inserted into pET22b vector to create the plasmid pET22b-SparsM. The pET22b-SparsM was subsequently transformed into the E. coli Transetta (DE3) or AW3110 ( $\triangle$ arsRBC). The His-tagged SpArsM was composed of 401 amino acids with a mass of $45 \mathrm{kDa}$. By carrying out multiple-sequence alignment of arsenite S-adenosylmethionine methyltransferase (ArsM) orthologs, we found that the $\mathrm{N}$-termini and $\mathrm{C}$-termini are not conserved, and an $\mathrm{N}$ - and C-terminally truncated derivative of ArsM from Tetrahymena pyriformis did not lose the ability to methylate As(III) (Ye et al., 2014). To verify whether the last 34 residues of SpArsM affected As(III) methylation, the truncated derivative of SpArsM (arsM34) was also constructed by using primers 5'-CATATGACTCCAGCTAAATCGGCAAATACTG-3' (NdeI site underlined) and 5'-CTCGAGACAATCAAATTCTTTAGCATC CTTTAG-3' (XhoI site underlined), resulting in a protein of 367 amino acids with a mass of $41 \mathrm{kDa}$. 


\subsection{Resistance assays in E. coli}

Arsenic resistance assays were performed as previously reported (Qin et al., 2006). Single colonies of E. coli AW3110 ( $\triangle$ arsRBC) bearing pET22b and pET22b-SparsM (full-length) were inoculated into $5 \mathrm{~mL} \mathrm{LB}$ medium containing $100 \mu \mathrm{g} / \mathrm{mL}$ Ampicillin and $30 \mu \mathrm{g} / \mathrm{mL}$ Chloramphenicol. After incubating at $37^{\circ} \mathrm{C}$ overnight with shaking at $180 \mathrm{r} / \mathrm{min}$, the late exponential phase cells were diluted 100-fold into $40 \mathrm{~mL}$ fresh LB medium containing the antibiotics, $0.3 \mathrm{mmol} / \mathrm{L}$ isopropyl $\beta$-D-1-thiogalactopyranoside (IPTG) and the indicated concentrations of As(III). Growth was monitored as optical density at $600 \mathrm{~nm}$ by using an ultravioletvisible spectrophotometer (Mapada).

\subsection{Arsenic methylation assays in $\mathbf{E}$. coli}

As(III) methylation was measured in E. coli AW3110 ( $\triangle$ arsRBC) bearing either plasmid pET22b-SparsM or empty vector pET22b. Cultures were grown overnight in LB medium containing $100 \mu \mathrm{g} / \mathrm{mL}$ Ampicillin and $30 \mu \mathrm{g} / \mathrm{mL}$ Chloramphenicol, then diluted 100 -folds into $5 \mathrm{~mL}$ fresh LB medium containing the corresponding antibiotics. Final concentrations of $0.3 \mathrm{mmol} / \mathrm{L}$ IPTG and $10 \mu \mathrm{mol} / \mathrm{L} \mathrm{As(III)} \mathrm{were} \mathrm{added}$ when cells reached exponential phase. Culture samples were centrifuged at 12,000 r/min for $1 \mathrm{~min}$ after $19 \mathrm{hr}$ incubation. The supernatants were filtrated through $0.22 \mu \mathrm{m}$ filters and then stored at $-20^{\circ} \mathrm{C}$ until As species were analyzed. Volatile As produced by SparsM was collected as described (Huang et al., 2012).

\subsection{Overexpression and purification of SpArsM}

Cells of Transetta (DE3) bearing pET22b-SparsM or pET22bSparsM34 were incubated at $37^{\circ} \mathrm{C}$ in $\mathrm{LB}$ media, $0.3 \mathrm{mmol} / \mathrm{L}$ IPTG was supplemented to induce expression of His-tagged SpArsM or SpArsM34 when $\mathrm{OD}_{600}$ reached 0.4-0.6. After continuing to incubate at $18^{\circ} \mathrm{C}$ overnight, cells were harvested by centrifugation $(6000 \mathrm{r} / \mathrm{min})$ at $4^{\circ} \mathrm{C}$ for $15 \mathrm{~min}$ and then suspended in buffer A $(20 \mathrm{mmol} / \mathrm{L}$ HEPES, $0.5 \mathrm{~mol} / \mathrm{L} \mathrm{NaCl}$, $3 \mathrm{mmol} / \mathrm{L}$ tris(2-carboxyethyl)phosphine (TCEP), pH 7.4). The cells were lysed by a single pass through a French-press at $10 \mathrm{MPa}$ and the lysate was centrifugated at 19,000 r/min for $45 \mathrm{~min}$ to remove cell debris and unbroken cells. The supernatant was filtrated through a $0.22 \mu \mathrm{m}$ filter and loaded onto a Ni-NTA agarose column (Qiagen) pre-equilibrated with buffer A. The column was washed by $100 \mathrm{~mL}$ buffer B (20 mmol/L HEPES, $0.5 \mathrm{~mol} / \mathrm{L} \mathrm{NaCl}, 20 \mathrm{mmol} / \mathrm{L}$ imidazole and $3 \mathrm{mmol} / \mathrm{L} \mathrm{TCEP}$, $\mathrm{pH}$ 7.0) and finally eluted with $15 \mathrm{~mL}$ buffer $\mathrm{C}(20 \mathrm{mmol} / \mathrm{L}$ HEPES, $0.5 \mathrm{~mol} / \mathrm{L} \mathrm{NaCl}, 500 \mathrm{mmol} / \mathrm{L}$ imidazole and $3 \mathrm{mmol} / \mathrm{L}$ TCEP, pH 7.0). Fractions containing SpArsM or SpArsM34 were concentrated using a 10-kDa-cutoff Amicon Ultrafilter (Millipore). Protein concentrations were determined by absorbance at $280 \mathrm{~nm}$. SpArsM and SpArsM34 proteins were checked by sodium-dodecyl-sulfate polyacrylamide gel electrophoresis (SDS-PAGE).

\subsection{As(III) methylation assay using purified SpArsM protein}

As(III) methylation in vitro assays with purified SpArsM or SpArsM34 were performed in buffer D $(50 \mathrm{mmol} / \mathrm{L}$ MOPS and
$125 \mathrm{mmol} / \mathrm{L} \mathrm{KCl}, \mathrm{pH}$ 7.5) containing $8 \mathrm{mmol} / \mathrm{L} \mathrm{GSH}, 1 \mathrm{mmol} / \mathrm{L}$ SAM, $5 \mu \mathrm{mol} / \mathrm{L}$ ArsM and $10 \mu \mathrm{mol} / \mathrm{L} \mathrm{As(III)} \mathrm{at} 37^{\circ} \mathrm{C}$ for $12 \mathrm{hr}$. The reactions were terminated by boiling for $5 \mathrm{~min}$. Some of the reaction solutions were oxidized by $30 \%(\mathrm{~V} / \mathrm{V}) \mathrm{H}_{2} \mathrm{O}_{2}$. The changes of As species during the reactions were monitored at indicated time $(0,0.5,1,3,6,12 \mathrm{hr})$.

\subsection{Preparation of SpArsM mutants}

Mutations of SpArsM were generated by site-directed mutagenesis of pET22b-SparsM using primers listed in Appendix A Table S1. Three single mutants of SpArsM (C59S, C186S, or C238S) were generated by changing codons for cysteine to serine. Each mutation was confirmed by sequencing. The plasmid vectors carrying different mutants were transformed into E. coli AW3110.

\subsection{Arsenic speciation analysis}

Arsenic speciation was analyzed by high-performance liquid chromatography-inductively coupled plasma mass spectrometry (HPLC-ICP-MS) (Agilent 1200 series and Agilent 7500 series, Agilent Technologies, USA) as previously described (Zhu et al., 2008). A Hamilton PRP-X100 10- $\mu \mathrm{m}$ anion-exchange column $\left(250 \times 4.1 \mathrm{~mm}^{2}\right)$ was used for chromatography. The mobile phase consisted of $10 \mathrm{mmol} / \mathrm{L}$ diammonium hydrogen phosphate $\left(\left(\mathrm{NH}_{4}\right)_{2} \mathrm{HPO}_{4}\right)$ and $10 \mathrm{mmol} / \mathrm{L}$ ammonium nitrate $\left(\mathrm{NH}_{4} \mathrm{NO}_{3}\right)$ and adjusted to $\mathrm{pH} 6.2$ with nitric acid, at a flow rate of $1.0 \mathrm{~mL} / \mathrm{min}$. The analytical method was validated through analyses of GBW10025-a spiral seaweed (certified [total As] = $0.22 \pm 0.03 \mathrm{mg} / \mathrm{kg}$, certified [total $\mathrm{Cr}$ ] $=1.50 \pm 0.13 \mathrm{mg} / \mathrm{kg}$, found [total As] $=0.26 \pm 0.03 \mathrm{mg} / \mathrm{kg}$, and [total $\mathrm{Cr}]=1.63 \pm 0.16 \mathrm{mg} / \mathrm{kg}$ ).

\section{Results}

\subsection{Arsenic methylation by S. platensis}

In order to investigate whether S. platensis is capable of As methylation under laboratory conditions, S. platensis was cultured in Zarrouk's medium under exposure to different concentrations of As(III) for 15 days and the As species in cells were determined. $\mathrm{As}(\mathrm{V})$ was the dominant intracellular species accounting for $64 \%$ to $86 \%$ of the total arsenic (Fig. 1). About $2 \%$ to $15 \%$ of the total arsenic were in the form of methylated arsenic species including $\mathrm{DMA}(\mathrm{V})$ and $\mathrm{MMA}(\mathrm{V})$ after exposure to the indicated concentrations of As(III). This showed that S. platensis was able to accumulate and methylate As. No volatile As was detected.

\subsection{Cloning of SparsM}

PCR cloning using total DNA extracts from S. platensis yielded one arsM gene, SparsM. SpArsM was 97\% identical to the putative product of arsM from Arthrospira platensis. Multiple-sequence alignment showed that SpArsM shared 39\%,33\%, and 35\% identity with the annotated arsenite mthyltransferase proteins in Methanosarcina mazei Go1 (GenBank accession no. WP_ 011032612.1), Rhodopseudomonas palustris CGA009 (GenBank accession no. WP_011159102), and Aspergillus fumigatus (GenBank accession no. XM_748062), respectively. It also exhibited lower 


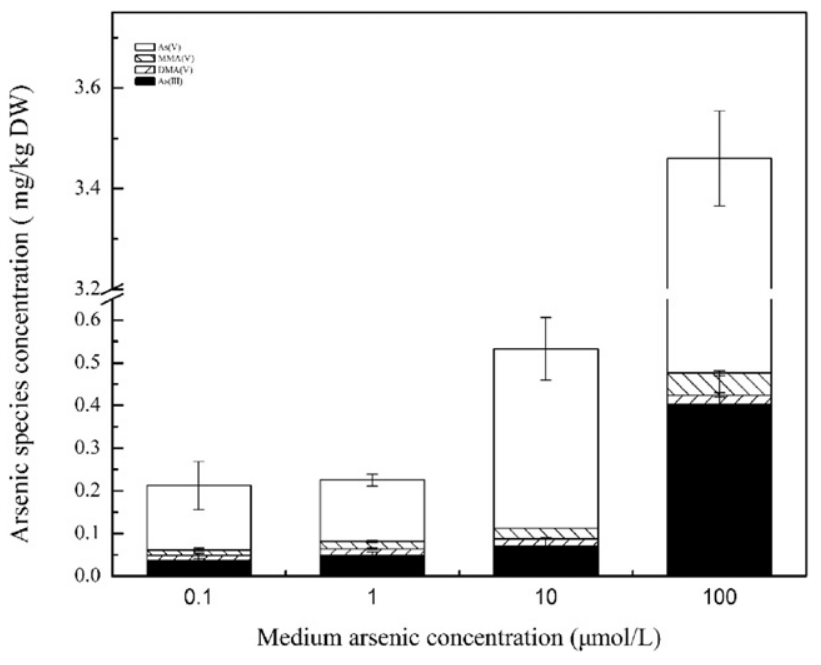

Fig. 1 - Water-soluble arsenic species in S. platensis after exposure to indicated concentrations of $\mathrm{As}$ (III) for 15 days, DW: dry weight.

identity to eukaryotic Cyanidioschyzon sp. 5508 ArsM (GenBank accession no. ACN39190), the AS3MT arsenite methyltransferases from human (Genbank accession no. AAI19639.2), and Danio rerio (GenBank accession no. NP_001034928.1) (32\%, 27\%, and 26\% identity, respectively). In addition, SpArsM only showed $26 \%$ and $27 \%$ identity with the two cyanobacterial Synechocystis sp. PCC 6803 and Nostoc sp. PCC 7120 ArsMs, respectively. The phylogenetic analysis (Appendix A Fig. S2) showed that the three cyanobacterial ArsMs belong to two different groups, the reason for the distant relationship between the three is not clear.

\subsection{SpArsM conferred resistance to As(III) in E. coli}

To examine whether SpArsM enhances As resistance, the SparsM gene was expressed in As-sensitive E. coli AW3110 ( $\triangle$ arsRBC) which had no orthologous arsM gene. At $70 \mu \mathrm{mol} / \mathrm{L}$ or $90 \mu \mathrm{mol} / \mathrm{L} \mathrm{As(III),} \mathrm{cells} \mathrm{bearing} \mathrm{the} \mathrm{SparsM} \mathrm{gene} \mathrm{grew} \mathrm{much}$ better than those bearing pET22b (Fig. 2), demonstrating that SpArsM conferred resistance to As(III). In addition, AW3110 bearing SparsM grew slightly better than the control in the absence of As(III).

\subsection{As(III) methylation by E. coli AW3110 expressing SparsM}

To test As(III) methylation activity of SpArsM in vivo, E. coli strain AW3110 bearing SparsM or pET22b was incubated with $10 \mu \mathrm{mol} / \mathrm{L}$ As(III) for $19 \mathrm{hr}$, and As species were analyzed (Fig. 3). Methylated arsenicals were not detected in the medium containing E. coli AW3110 bearing pET22b. Meanwhile, we found that the increase of $\mathrm{DMA}(\mathrm{V})$ concentration was coincident with the decrease of As(III) in the medium containing E. coli AW3110 expressing SparsM. DMA(V) was the predominate product, accounting for $84 \%$ of total As. In addition, small amounts of MMA(V) were also detected in the medium, while no volatilized As was detected no matter what concentration of As(III) $(1,10,70$, or $90 \mu \mathrm{mol} / \mathrm{L})$ was added into the media (data was not shown). This result showed SpArsM had the ability of

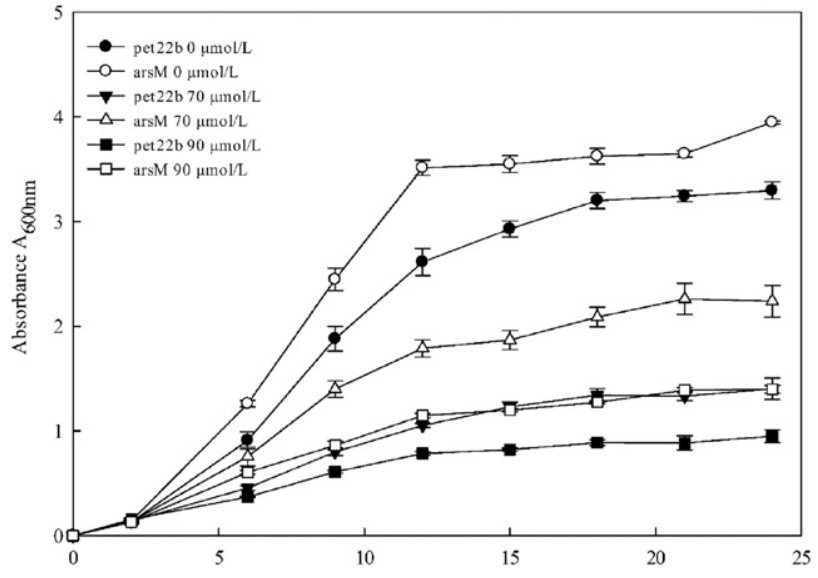

Fig. 2 - Expression of SparsM conferred arsenite resistance in E. coli AW3110. Cellular growth was monitored as absorbance at $600 \mathrm{~nm}$ at indicated time points. The data represent the average of 3 replicate cultures.

As(III) methylation, but the end product was DMA(V), not TMA(III).

\subsection{In vitro As(III) methylation using purified SpArsM and} SpArsM34

To further elucidate the function of SpArsM and SpArsM34 in As(III) methylation, both SparsM and SparsM34 were expressed in E. coli Transetta and purified in a single step by Ni(II)-nitrilotriacetic acid (NTA) chromatography. Purified SpArsM protein showed As(III) methylation activity at $\mathrm{pH}$ values between 2.5 and 9.5, with the highest As(III) methylation activity being observed at pH 7.5 (Appendix A Fig. S3B). Furthermore, SpArsM could convert almost all As(III) to $\mathrm{DMA}(\mathrm{V})$ at $16^{\circ} \mathrm{C}$ and $37^{\circ} \mathrm{C}$, while the rate of As(III) methylation was reduced at $60^{\circ} \mathrm{C}$, resulting in more $\mathrm{MMA}(\mathrm{V})$ than $\mathrm{DMA}(\mathrm{V})$ being produced (Appendix A Fig. S3C). The time-dependent methylation products generated by SpArsM under optimal reaction condition $\left(37^{\circ} \mathrm{C}\right.$ and $\left.\mathrm{pH} 7.5\right)$ were also analyzed (Fig. 4A). As(III) methylation to DMA(V) was observed within

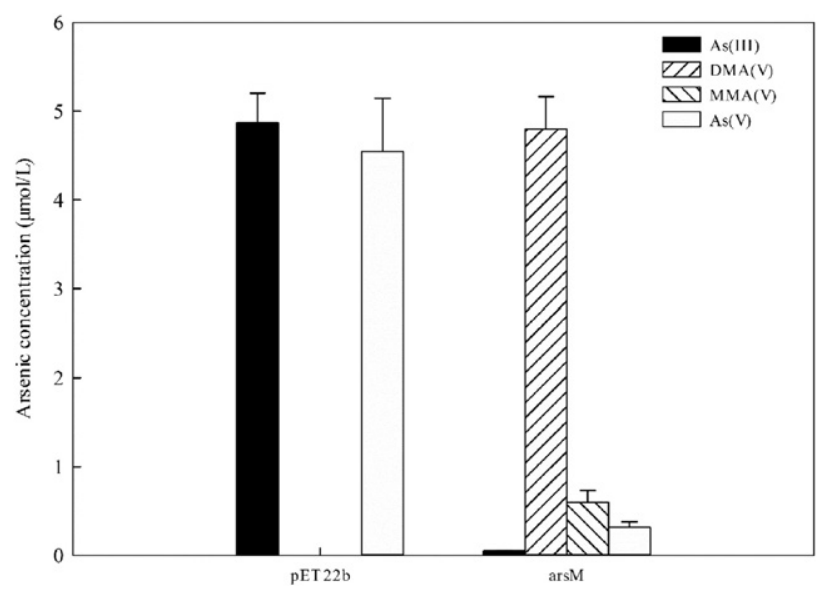

Fig. 3 - The As species in the medium incubating E. coli AW3110 expressing SparsM for $19 \mathrm{hr}$ at $37^{\circ} \mathrm{C}$. 

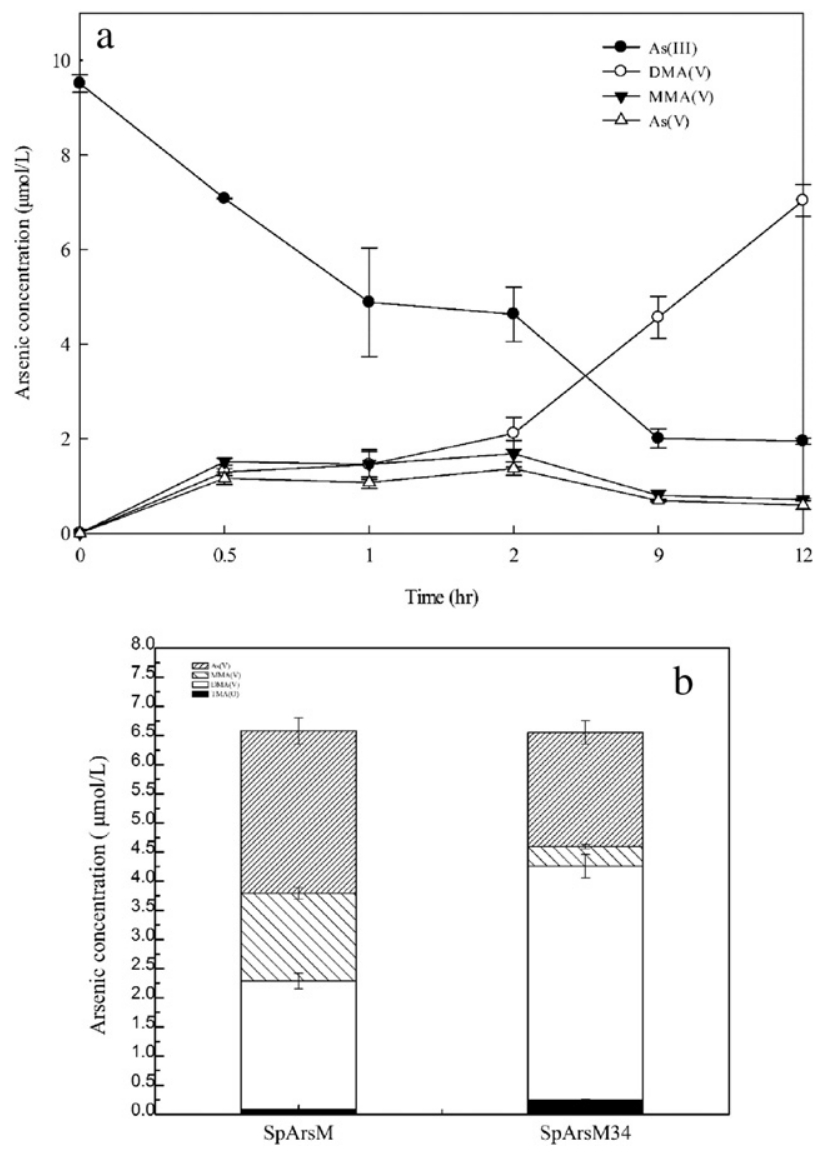

Fig. 4 - As(III) methylation by purified SpArsM and SpArsM34. (a) Time course of As(III) methylation by purified SpArsM. (b) As species of As(III) methylation by purified SpArsM and SpArsM34 in MOPS buffer (pH 7.5) at $37^{\circ} \mathrm{C}$ for $12 \mathrm{hr}$.

$0.5 \mathrm{hr}$. In order to verify whether the C-terminal residues of SpArsM would have an impact on As(III) methylation, in vitro catalysis of the truncated SpArsM derivative (SpArsM34) was also performed under the same condition. Results showed SpArsM34 was also able to methylate As(III) to DMA(V) (Fig. 4B), and the catalytic activity of SpArsM34 was higher than SpArsM.

\subsection{Conserved cysteine residues Cys59, Cys186, and Cys238 of SpArsM}

Conserved cysteine residues in As(III) methyltransferases are critical in catalyzing As methylation (Marapakala et al., 2012; Qin et al., 2006; Song et al., 2011). The amino acid sequence of SpArsM contains 16 cysteine residues, and Cys59, Cys186, and Cys238 are the conserved cysteines as detected by multiple alignment. Herein, the three cysteine residues were individually altered to serine by site-directed mutagenesis to create C59S, C186S, and C238S derivatives. In order to test whether the three cysteines are involved in As(III) methylation, the in vivo As methylation ability of these three mutants expressed in E. coli AW3110 were studied. All of the three SpArsM mutants completely abolished the As(III) methylation capability, and no methylated arsenic species were detected in the medium (Fig. 5). These results indicated that each of the three conserved cysteine residues was required for As(III) methylation.

\section{Discussion}

Arsenic is ubiquitous in aquatic environments where various kinds of cyanobacteria are distributed (Qin et al., 2006, 2009; Zhang et al., 2013), so cyanobacteria have evolved several ways to detoxify As in order to survive in the environment (Aurilio et al., 1994; Knauer et al., 1999). In this study, our results showed that $S$. platensis could grow at high concentrations of the toxic metalloid and had the capability of As(III) accumulation and biotransformation. In general, As(III) is detoxified directly by efflux out of cells, oxidation to $\mathrm{As}(\mathrm{V})$, or methylation to methylated As species (Bentley and Chasteen, 2002). Here, we found that these parallel pathways of As(III) detoxification exist in S. platensis. When S. platensis was exposed to As(III), the main As species was As(V) in S. platensis which indicated As(III) oxidation may be the primary As(III) detoxification pathway. This is similar to the response of other cyanobacteria, such as Synechocystis sp. PCC6803 (Yin et al., 2011), which oxidizes As(III) to As(V) for detoxification. Moreover, S. platensis showed the ability of As(III) methylation after a long-time As(III) exposure. As methylation is one of the crucial processes governing the fate of As in the environment. It is necessary to understand the mechanism of the different As methyltransferases. The As methylation pathway has been studied for a long time. However, the ArsMs responsible for As(III) methylation were identified and characterized only in recent years (Lin et al., 2002). We found an ArsM orthologue from S. platensis, and verified that it is responsible for As(III) biomethylation. Both E. coli bearing the SparsM gene and purified SpArsM protein conferred the ability to methylate As(III). These results clearly suggest that ArsM-mediated As

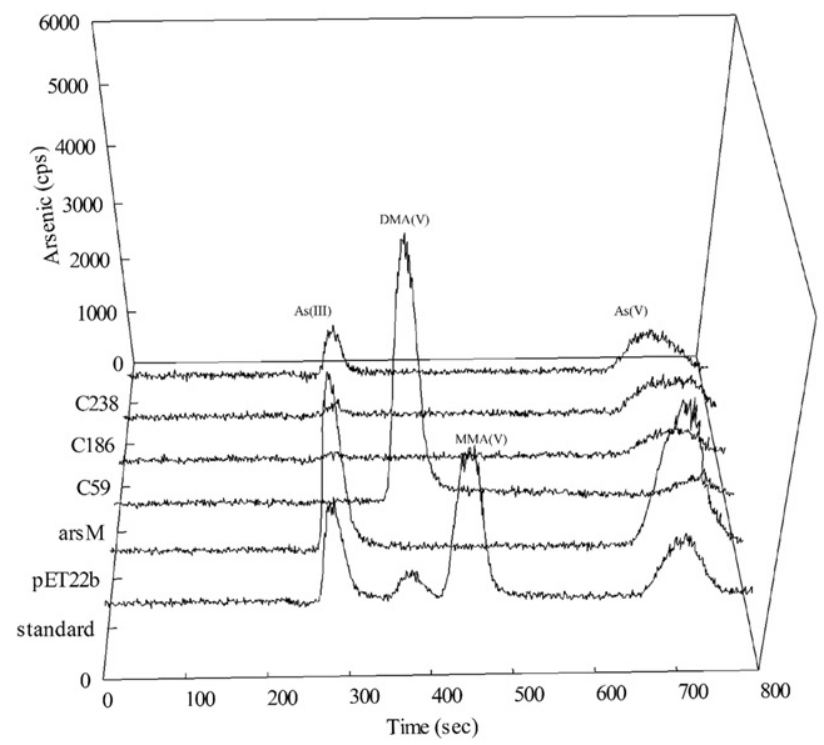

Fig. 5 - As(III) methylation in vivo assay of E. coli AW3110 expressing wild-type and mutants of SpArsM in the presence of $10 \mu \mathrm{mol} / \mathrm{L} \mathrm{As(III)} \mathrm{at} 37^{\circ} \mathrm{C}$ for $24 \mathrm{hr}$. 
methylation in S. platensis is a possible detoxification mechanism.

Although As biomethylation has been widely studied in bacteria, archaea, and fungi and the end product always was the volatilizable TMA(III) (Challenger, 1945; Hayakawa et al., 2005; Marapakala et al., 2012), we found S. platensis cannot generate TMA(III), and As(III) methylation of SpArsM ended with $\mathrm{DMA}(\mathrm{V})$ as the final product in both in vivo and vitro assays. This is similar to results in zebra fish (Hamdi et al., 2012). As(III) undergoes sequential methylations producing mono-, di-, and trimethylated species by ArsM, and the last methylation reaction to generate TMA(III) from DMA(V) is proposed to be the rate-limiting step (Marapakala et al., 2012). Herein, we suggest that the last reaction of As(III) methylation was not catalyzed by SpArsM, and DMA(V) might be used to produce arsenosugars or arsenolipids in S. platensis. According to the result of multiple-sequence alignments, SpArsM exhibited low similarity to other ArsMs from cyanobacteria with the sequence identity to Synechocystis sp. PCC 6803 and Nostoc sp. PCC 7120 ArsM being only $26 \%$ and $27 \%$, respectively. The huge difference between the sequences may explain the difference in methylation products. In addition, As(III) was seen to be transformed to $\mathrm{DMA}(\mathrm{V})$ within $30 \mathrm{~min}$, and the catalytic rate of SpArsM was much faster than that of other ArsMs (Qin et al., 2009; Wang et al., 2014a; Zhang et al., 2015). Meanwhile, the intermediate MMA(III) and MMA(V) were absent in the in vitro enzyme activity assays when the reaction was performed at optimal conditions, and only MMA(V) was observed when the enzyme was impaired, for example under higher or lower temperature. This result may possibly be due to MMA strongly binding to SpArsM or the methylation process from MMA to DMA was so quick that little MMA was released into the reaction medium.

$\mathrm{N}$ - or C-terminal residues of enzymes related to arsenic resistance are usually not required for their functions, while cysteine residues are often conserved and play an important role in the structure and function of the enzymes (Dheeman et al., 2014; Fomenko et al., 2008; Marapakala et al., 2012). The multiple-sequence alignment of ArsM illustrates that three cysteine residues are highly conserved and were shown to be involved in As(III) binding and catalysis of As methylation by site-directed mutagenesis (Ajees et al., 2012; Dheeman et al., 2014; Marapakala et al., 2012, 2015). Herein, we constructed a C-terminal truncated SpArsM and three cysteine mutants (C59S, C186S, C238S) to investigate whether deleting or mutating these residues had an effect on the function of SpArsM. Our results demonstrate that SpArsM34 still showed the ability of As methylation, and this result was not surprising because other truncated ArsMs from Tetrahymena pyriformis (30TpyarsM8) (Ye et al., 2014) and Cyanidioschyzon sp. 5508 (Ajees et al., 2012) did not lose their ability to methylate As(III). In our study, the catalytic rate of SpArsM34 increased compared to the full-length SpArsM. This result indicated that the truncation of amino acids may facilitate the stability of protein structure which results in an increase of enzyme activity in vitro (Beckstead et al., 2005). Moreover, neither of the single cysteine mutants of SpArsM retained the ability to methylate As(III), which indicated that Cys59, Cys186, and Cys238 of SpArsM are essential in maintaining the function. The three cysteines are conserved in all of the ArsMs ranged from bacteria to humans, and more work should be done to elucidate the evolution of ArsM.

In conclusion, our results clearly showed that S. platensis was able to accumulate and biotransform As(III), and the molecular mechanism of As methylation by SpArsM was also elucidated. S. platensis is not only a crucial part of the food chain in aquatic environments but also can be a direct source of As exposure for humans by being used as a health supplement. The potential risk of S. platensis regarding arsenic accumulation may have been overlooked. Our results may help understand the role of S. platensis in As biogeochemistry in the environment, and could be further used to evaluate the risk of S. platensis in the food chain and to explore the possibility to be applied as a bioremediation material to remove As from As-contaminated water.

\section{Acknowledgments}

This work was supported by the Natural Science Foundation of Fujian Province (No. 2014J01141), the National Natural Science Foundation of China (No. 31270161) and the Fujian-Taiwan Joint Innovative Center for Germplasm Resources and Cultivation of Crop (No. Fujian 2011 Program, [2015]75).

\section{Appendix A. Supplementary data}

Supplementary data to this article can be found online at http://dx.doi.org/10.1016/j.jes.2016.06.013.

\section{R E F E R E N C E S}

Ajees, A.A., Marapakala, K., Packianathan, C., Sankaran, B., Rosen, B.P., 2012. Structure of an As(III) S-adenosylmethionine methyltransferase: insights into the mechanism of arsenic biotransformation. Biochemistry 51 (27), 5476-5485.

Armendariz, A.L., Talano, M.A., Oller, A.L.W., Medina, M.I., Agostini, E., 2015. Effect of arsenic on tolerance mechanisms of two plant growth-promoting bacteria used as biological inoculants. J. Environ. Sci. 33 (7), 203-210.

Aurilio, A.C., Mason, R.P., Hemond, H.F., 1994. Speciation and fate of arsenic in three lakes of the Aberjona watershed. Environ. Sci. Technol. 28 (4), 577-585.

Babadzhanov, A., Abdusamatova, N., Yusupova, F., Faizullaeva, N., Mezhlumyan, L., Malikova, M.K., 2004. Chemical composition of Spirulina platensis cultivated in Uzbekistan. Chem. Nat. Compd. 40 (3), 276-279.

Beckstead, J.A., Block, B.L., Bielicki, J.K., Kay, C.M., Oda, M.N., Ryan, R.O., 2005. Combined N- and C-terminal truncation of human apolipoprotein A-I yields a folded, functional central domain. Biochemistry 44 (11), 4591-4599.

Bentley, R., Chasteen, T.G., 2002. Microbial methylation of metalloids: arsenic, antimony, and bismuth. Microbiol. Mol. Biol. Rev. 66 (2), 250-271.

Brammer, H., Ravenscroft, P., 2009. Arsenic in groundwater: a threat to sustainable agriculture in South and South-east Asia. Environ. Int. 35 (3), 647-654.

Campanella, L., Crescentini, G., Avino, P., Moauro, A., 1998. Determination of macrominerals and trace elements in the alga Spirulina platensis. Analusis 26 (5), 210-214. 
Carlin, A., Shi, W.P., Dey, S., Rosen, B.P., 1995. The Ars Operon of Escherichia coli confers arsenical and antimonial resistance. J. Bacteriol. 177 (4), 981-986.

Challenger, F., 1945. Biological methylation. Chem. Rev. 36 (3), 315-361.

Christen, K., 2001. The arsenic threat worsens. Environ. Sci. Technol. 35 (13), 286A-291A.

Ciferri, O., 1983. Spirulina, the edible microorganism. Microbiol. Rev. 47 (4), 551-578.

Cullen, W.R., Reimer, K.J., 1989. Arsenic speciation in the environment. Chem. Rev. 89 (4), 713-764.

Del Castillo, B.D., 1956. The Discovery and Conquest of Mexico. Octagon Books, New York, p. 1970.

Dheeman, D.S., Packianathan, C., Pillai, J.K., Rosen, B.P., 2014. Pathway of human AS3MT arsenic methylation. Chem. Res. Toxicol. 27 (11), 1979-1989.

Disyawongs, G., 2002. Accumulation of copper, mercury, and lead in Spirulina platensis studied in Zarrouk's medium. J. KMITNB 12 (4), 33-35.

Doshi, H., Ray, A., Kothari, I., 2009. Live and dead Spirulina sp. to remove arsenic (V) from water. Int. J. Phytoremediation 11 (1), 53-64.

Fomenko, D.E., Marino, S.M., Gladyshev, V.N., 2008. Functional diversity of cysteine residues in proteins and unique features of catalytic redox-active cysteines in thiol oxidoreductases. Mol. Cell 26 (3), 228-235.

Hamdi, M., Yoshinaga, M., Packianathan, C., Qin, J., Hallauer, J., McDermott, J.R., et al., 2012. Identification of an S-adenosylmethionine (SAM) dependent arsenic methyltransferase in Danio rerio. Toxicol. Appl. Pharmacol. 262 (2), 185-193.

Hayakawa, T., Kobayashi, Y., Cui, X., Hirano, S., 2005. A new metabolic pathway of arsenite: arsenic-glutathione complexes are substrates for human arsenic methyltransferase Cyt19. Arch. Toxicol. 79 (4), 183-191.

Huang, H., Jia, Y., Sun, G.X., Zhu, Y.G., 2012. Arsenic speciation and volatilization from flooded paddy soils amended with different organic matters. Environ. Sci. Technol. 46 (4), 2163-2168.

Knauer, K., Behra, R., Hemond, H., 1999. Toxicity of inorganic and methylated arsenic to algal communities from lakes along an arsenic contamination gradient. Aquat. Toxicol. 46 (3), 221-230.

Lin, S., Shi, Q., Nix, F.B., Styblo, M., Beck, M.A., Herbin-Davis, K.M., et al., 2002. A novel S-adenosyl-l-methionine: arsenic (III) methyltransferase from rat liver cytosol. J. Biol. Chem. 277 (13), 10795-10803.

Lodi, A., Soletto, D., Solisio, C., Converti, A., 2008. Chromium (III) removal by Spirulina platensis biomass. Chem. Eng. J. 136 (2), 151-155.

Maher, W.A., Foster, S.D., Taylor, A.M., Krikowa, F., Duncan, E.G., Chariton, A.A., 2011. Arsenic distribution and species in two Zostera capricorni seagrass ecosystems, New South Wales, Australia. Environ. Chem. 8 (1), 9-18.

Marapakala, K., Packianathan, C., Ajees, A.A., Dheeman, D.S., Sankaran, B., Kandavelu, P., et al., 2015. A disulfide-bond cascade mechanism for arsenic (III) S-adenosylmethionine methyltransferase. Acta Crystallogr. D 71 (3), 505-515.

Marapakala, K., Qin, J., Rosen, B.P., 2012. Identification of catalytic residues in the As(III) S-adenosylmethionine methyltransferase. Biochemistry 51 (5), 944-951.

Meliker, J.R., Wahl, R.L., Cameron, L.L., Nriagu, J.O., 2007. Arsenic in drinking water and cerebrovascular disease, diabetes mellitus, and kidney disease in Michigan: a standardized mortality ratio analysis. Environ. Health 6 (4), 1-11.

Paez-Espino, D., Tamames, J., de Lorenzo, V., Canovas, D., 2009. Microbial responses to environmental arsenic. Biometals 22 (1), 117-130.

Pan, W.S., Wu, C., Xue, S.G., Hartley, W., 2014. Arsenic dynamics in the rhizosphere and its sequestration on rice roots as affected by root oxidation. J. Environ. Sci. 26 (4), 892-899.
Qin, J., Lehr, C.R., Yuan, C., Le, X.C., McDermott, T.R., Rosen, B.P., 2009. Biotransformation of arsenic by a Yellowstone thermoacidophilic eukaryotic alga. Proc. Natl. Acad. Sci. U. S. A. 106 (13), 5213-5217.

Qin, J., Rosen, B.P., Zhang, Y., Wang, G., Franke, S., Rensing, C., 2006. Arsenic detoxification and evolution of trimethylarsine gas by a microbial arsenite S-adenosylmethionine methyltransferase. Proc. Natl. Acad. Sci. U. S. A. 103 (7), 2075-2080.

Rahman, M.A., Hasegawa, H., Lim, R.P., 2012. Bioaccumulation, biotransformation and trophic transfer of arsenic in the aquatic food chain. Environ. Res. 116 (6), 118-135.

Richmond, A., Hu, Q., 2013. Handbook of Microalgal Culture: Applied Phycology and Biotechnology. John Wiley \& Sons, Place.

Seshadri, C., Thomas, S., 1979. Mass culture of Spirulina using low-cost nutrients. Biotechnol. Lett. 1 (7), 287-291.

Smedley, P., Kinniburgh, D., 2002. A review of the source, behaviour and distribution of arsenic in natural waters. Appl. Geochem. 17 (5), 517-568.

Solisio, C., Lodi, A., Torre, P., Converti, A., Del Borghi, M., 2006. Copper removal by dry and re-hydrated biomass of Spirulina platensis. Bioresour. Technol. 97 (14), 1756-1760.

Song, X., Geng, Z., Li, X., Zhao, Q., Hu, X., Zhang, X., et al., 2011. Functional and structural evaluation of cysteine residues in the human arsenic (+3 oxidation state) methyltransferase (hAS3MT). Biochimie 93 (2), 369-375.

Styblo, M., Drobna, Z., Jaspers, I., Lin, S., Thomas, D.J., 2002. The role of biomethylation in toxicity and carcinogenicity of arsenic: a research update. Environ. Health Perspect. 110 (Suppl. 5), 767-771.

Vonshak, A., 1997. Spirulina platensis Arthrospira: Physiology, Cell-Biology and Biotechnology. CRC Press, Place.

Wang, P.-P., Sun, G.-X., Zhu, Y.-G., 2014a. Identification and characterization of arsenite methyltransferase from an archaeon, Methanosarcina acetivorans C2A. Environ. Sci. Technol. 48 (21), 12706-12713.

Wang, P.P., Sun, G.X., Jia, Y., Meharg, A.A., Zhu, Y.G., 2014b. A review on completing arsenic biogeochemical cycle: microbial volatilization of arsines in environment. J. Environ. Sci. 26 (2), 371-381.

Yan, Y., Ye, J., Xue, X.-M., Zhu, Y.-G., 2015. Arsenic demethylation by a C.As lyase in cyanobacterium Nostoc sp. PCC 7120. Environ. Sci. Technol. 49 (24), 14350-14358.

Ye, J., Chang, Y., Yan, Y., Xiong, J., Xue, X.M., Yuan, D., et al., 2014. Identification and characterization of the arsenite methyltransferase from a protozoan, Tetrahymena pyriformis. Aquat. Toxicol. 149 (22), 50-57.

Ye, J., Rensing, C., Rosen, B.P., Zhu, Y.G., 2012. Arsenic biomethylation by photosynthetic organisms. Trends Plant Sci. 17 (3), 155-162.

Yin, X.-X., Wang, L.H., Bai, R., Huang, H., Sun, G.-X., 2011. Accumulation and transformation of arsenic in the blue-green alga Synechocysis sp. PCC6803. Water Air Soil Pollut. 223 (3), 1183-1190.

Zhang, J., Cao, T., Tang, Z., Shen, Q., Rosen, B.P., Zhao, F.-J., 2015. Arsenic methylation and volatilization by arsenite S-adenosylmethionine methyltransferase in Pseudomonas alcaligenes NBRC14159. Appl. Environ. Microbiol. 81 (8), 2852-2860.

Zhang, S.Y., Sun, G.X., Yin, X.X., Rensing, C., Zhu, Y.G., 2013. Biomethylation and volatilization of arsenic by the marine microalgae Ostreococcus tauri. Chemosphere 93 (1), 47-53.

Zhu, Y.-G., Sun, G.-X., Lei, M., Teng, M., Liu, Y.-X., Chen, N.-C., et al., 2008. High percentage inorganic arsenic content of mining impacted and nonimpacted Chinese rice. Environ. Sci. Technol. 42 (13), 5008-5013. 